\title{
Characteristics of uveitic glaucoma and evaluation of its surgical treatment
}

Clinical Ophthalmology

26 November 2014

Number of times this article has been viewed

\section{Ai Shimizu \\ Kazuichi Maruyama \\ Yu Yokoyama \\ Satoru Tsuda \\ Morin Ryu \\ Toru Nakazawa}

Department of Ophthalmology, Tohoku University Graduate School of Medicine, Sendai, Japan
Correspondence: Toru Nakazawa

Department of Ophthalmology and Visual Science, Tohoku University Graduate

School of Medicine, I-I Seiryo-cho,

Aoba-ku, Sendai, Japan

Tel +8I $227 \mid 77294$

Fax +81227177298

Email ntoru@oph.med.tohoku.ac.jp
Purpose: To investigate the characteristics of uveitic glaucoma (UG) and evaluate surgical treatments.

Methods: This study examined a retrospective, nonrandomized comparative interventional case series of 105 UG patients (141 eyes) followed between April 1, 2001 and July 30, 2014 at the outpatient clinic of Tohoku University Hospital. The study group included 47 patients (47 eyes) who underwent glaucoma surgery: trabeculectomy, trabeculotomy, and trabectome surgery. The analysis used Kaplan-Meier life tables, with surgical failure defined as intraocular pressure $\geqq 21 \mathrm{mmHg}$ or the need for additional glaucoma surgery.

Results: UG patients represented $9.73 \%$ of our database of glaucoma patients. The mean follow-up period was $40.32 \pm 32.53$ months. Seventy-one patients had granulomatous uveitis $(67.62 \%)$ and 34 had nongranulomatous uveitis $(32.38 \%)$. The causes of uveitis included sarcoidosis $(n=25)$, Behçet's disease $(n=11)$, Vogt-Koyanagi-Harada disease $(n=9)$, PosnerSchlossman syndrome $(n=12)$, herpes simplex virus infectious uveitis $(n=7)$, acute anterior uveitis $(n=5)$, intermediate uveitis $(n=4)$, scleritis $(n=4)$, inflammatory bowel disease $(n=4)$, varicella zoster virus uveitis $(n=2)$, and others $(n=6)$. An additional 16 patients were diagnosed with idiopathic UG. Surgical success rates were $82.86 \%$ for trabeculectomy, $62.50 \%$ for trabeculotomy, and $75.00 \%$ for trabectome. Significant risk factors for surgical failure included male sex $(P=0.02)$, age less than 45 years $(P=0.0009)$, nongranulomatous uveitis $(P=0.04)$, and postoperative inflammation $(P=0.01)$.

Conclusion: Young male patients with nongranulomatous uveitis had a significant risk of surgical failure. Moreover, prolonged postoperative inflammation created a susceptibility to surgical failure, indicating the importance of postoperative inflammation reduction.

Keywords: uveitic glaucoma, sarcoidosis, Behçet's disease

\section{Introduction}

Although uveitis possesses many clinical features, transient high intraocular pressure (IOP) is commonly seen in practice. Pathological IOP elevation occurs in $20 \%-40 \%$ of uveitis patients, and $10 \%-40 \%$ of patients are treated for consecutive secondary glaucoma. ${ }^{1-4}$ The first choice for IOP control in these patients is medical ocular hypotensive therapy. The underlying mechanism of increased IOP in uveitis is known to involve disruptions in the ciliary process and blood-aqueous barrier, which allow plasma protein and inflammatory-mediated cells, such as neutrophils and T-cells, to gain access to the anterior chamber (AC), causing a subsequent increase in outflow resistance. ${ }^{1-4}$ Moreover, mechanical outflow disorders, such as peripheral anterior synechiae (PAS), which are caused by the formation of granulomas, can cause structural changes in the aqueous outflow pathway. These disorders can lead to secondary glaucoma requiring advanced surgical treatment, which can be challenging in these 
patients because of the increased risk of postoperative inflammation and subsequent surgical failure.

Early, accurate diagnosis of uveitis is therefore essential for the timely initiation of appropriate therapies and the achievement of successful clinical outcomes for uveitis patients. The incidence and the clinical appearance of uveitic glaucoma (UG) differ according to the etiology of the disease, leading to a wide variety of UG phenotypes clinically. The etiology of uveitis is also known to vary among different ethnicities, and even among regions in the same country. ${ }^{2}$ One example of this variation is the northern regions of Japan. Despite this wide variability in UG, and the current supposition that UG patients represent $5 \%-15 \%$ of all glaucoma patients, ${ }^{5-7}$ there are only a few current discussions of UG, despite its inclusion in glaucoma classification guidelines. Thus, in order to more precisely determine the clinical features of UG and their impact on clinical outcomes, the present study retrospectively investigated a group of UG patients at a single institution, Tohoku University, in northern Japan.

\section{Materials and methods}

\section{Patients}

We retrospectively examined the medical records of 1,079 glaucoma patients who were followed between April 1, 2001 and July 30, 2014 at the outpatient clinic of Tohoku University Hospital. The study protocol was approved by the Institutional Review Board of Tohoku University Hospital. Secondary glaucoma, including UG, was diagnosed by elevated IOP $(>21 \mathrm{mmHg})$ or the use of IOP-controlling medication, as defined in The Japan Glaucoma Society Guidelines for Glaucoma (3rd edition). ${ }^{8}$

We identified 105 UG patients (141 eyes), all diagnosed by uveitis specialists, who were divided into granulomatous and nongranulomatous groups according to the standards of the International Uveitis Study Group, including later modifications. ${ }^{9} 10$ All patients underwent treatment for UG for more than 3 months.

\section{Measurement of clinical outcomes}

Clinical characteristics were determined from patient's medical records, including age, sex, best-corrected visual acuity (BCVA), pretreatment IOP, pretreatment intraocular inflammation, pretreatment presence and extent of PAS, consecutive use of topical or systemic corticosteroid, number of acetazolamide tablets (each tablet contained $250 \mathrm{mg}$ of acetazolamide), and number of eye drops prescribed for ocular hypotensive therapy. Decimal visual acuity was converted to the minimum angle of resolution (logMAR). Low-vision patients were assigned decimal equivalents as follows: counting fingers $=0.00500$, hand motion $=0.00250$, light perception $=0.00125$, and no light perception $=0.00010 .{ }^{11}$ Intraocular inflammation was assessed with slit-lamp microscopy. Cells and flaring in the AC were scored using a semiquantitative scoring system with six grades: 0 , trace, $1+$, $2+, 3+$, and $4+.{ }^{12}$ Visual field results were excluded, as they can be unrepresentative of glaucoma progress in UG patients because of retinal and choroidal involvement.

\section{Measurement of surgical outcomes}

Surgical outcome and complications, as well as postoperative IOP and inflammation, were analyzed in detail in the patients who underwent glaucoma surgery, including trabeculectomy, trabeculotomy, and trabectome surgery, performed with or without cataract surgery. All patients were followed at Tohoku University for at least 1 year. Patients with 1) previous glaucoma surgery; 2) intraocular surgery within the previous 3 months; or 3) UG-related neovascularization were excluded. If both eyes underwent surgery, the eye that was treated first was examined. Postoperative examinations were performed at 2 weeks, 1 month, and 2 months, every quarter of 2 years, and semiannually thereafter. Surgical failure was defined as IOP more than $21 \mathrm{mmHg}$ in two consecutive visits, with or without eye drops prescribed for ocular hypotensive therapy, or by the need for additional glaucoma surgery or secondary procedures, such as needling. Postoperative inflammation was defined as cells or flaring graded $1+$ from 2 weeks to 3 months after surgery. Postoperative complications included severe hyphema in the AC, bleb leakage, choroidal detachment, cataract progression requiring surgery, hypotonic maculopathy, and blebitis/endophthalmitis.

\section{Surgical procedures}

Trabeculectomy was performed with a modified Cairns's technique. ${ }^{13}$ Mitomycin C (MMC) was applied intraoperatively to the scleral flap and under the conjunctiva. ${ }^{14}$ A conjunctival incision was then made using a fornix- or limbal-based procedure. After the creation of the scleral flap, sponges soaked in $0.4 \mathrm{mg} / \mathrm{mL}$ MMC were applied to the posterior surface of the conjunctiva, Tenon's capsule, the adjacent episcleral tissue, and the scleral flap for 5 minutes, followed by irrigation with balanced saline solution. A trabecular block was excised to create a fistula in the AC, and peripheral iridectomy was then performed. The scleral flap was closed with 10-0 nylon sutures, and the conjunctival flap was sutured with 10-0 nylon. Informed consent was obtained before for all procedures. 
Trabeculotomy was performed with a similar method as trabeculectomy until MMC irrigation was performed. In trabeculotomy, deep lamellar layers of the sclera were removed and deroofing of Schlemm's channel was performed. Two trabeculotomes were then placed through Schlemm's channel into the AC, lateral to the sclerectomy, to prevent vitreous prolapse. ${ }^{14,15} \mathrm{~A}$ scleral flap and a conjunctival flap were then created with a method similar to trabeculectomy.

Trabectome surgery was performed as follows: a $1.7 \mathrm{~mm}$ temporal corneal incision was made close to the limbus, parallel to the iris, and $0.1 \mathrm{~mL}$ of $1 \%$ lidocaine was injected into the $\mathrm{AC}$ as an anesthetic. Next, the trabectome handpiece was inserted into the $\mathrm{AC}$ and infusion begun. The handpiece was then moved further into the AC until its footplate penetrated the nasal meshwork. The surgeon then proceeded to cauterize the meshwork by moving the trabectome handpiece approximately $60^{\circ}$ superiorly and $60^{\circ}$ inferiorly.

\section{Statistical analysis}

Analysis was performed with the JMP version 10 statistical package (SAS Institute Inc., Cary, NC, USA). Evaluations of continuous variables were made with the Mann-Whitney $U$-test. Categorical variables were evaluated with the $\chi^{2}$ test. Kaplan-Meier life tables were used to calculate the surgical success rate. Statistical comparisons of the two groups were made with the log-rank test. $P<0.05$ was considered statistically significant. Data are shown as mean \pm SD.

\section{Results}

\section{UG patient characteristics}

The characteristics of the UG patients are shown in Table 1. UG was found in 105 (9.73\%) patients in our database, with 71 cases of granulomatous uveitis $(67.62 \%)$ and 34 cases of nongranulomatous uveitis (32.38\%). Uveitic causes of UG included sarcoidosis $(\mathrm{n}=25)$, Behçet's disease $(\mathrm{n}=11)$, Vogt-Koyanagi-Harada (VKH) disease $(n=9)$, PosnerSchlossman (P-S) syndrome ( $\mathrm{n}=12)$, herpes simplex virus infectious uveitis $(n=7)$, acute anterior uveitis $(n=5)$, intermediate uveitis $(n=4)$, scleritis $(n=4)$, inflammatory bowel disease $(n=4)$, varicella zoster virus uveitis $(n=2)$, and others $(\mathrm{n}=6)$. A further 16 patients were diagnosed with idiopathic UG lacking the characteristic of clinical features of any recognized uveitic entity, or not attributable to a specific systemic disease. There were significantly fewer male patients in the sarcoidosis group $(P=0.03)$ and patients with a significantly lower age in the acute anterior uveitis group $(P<0.05)$.

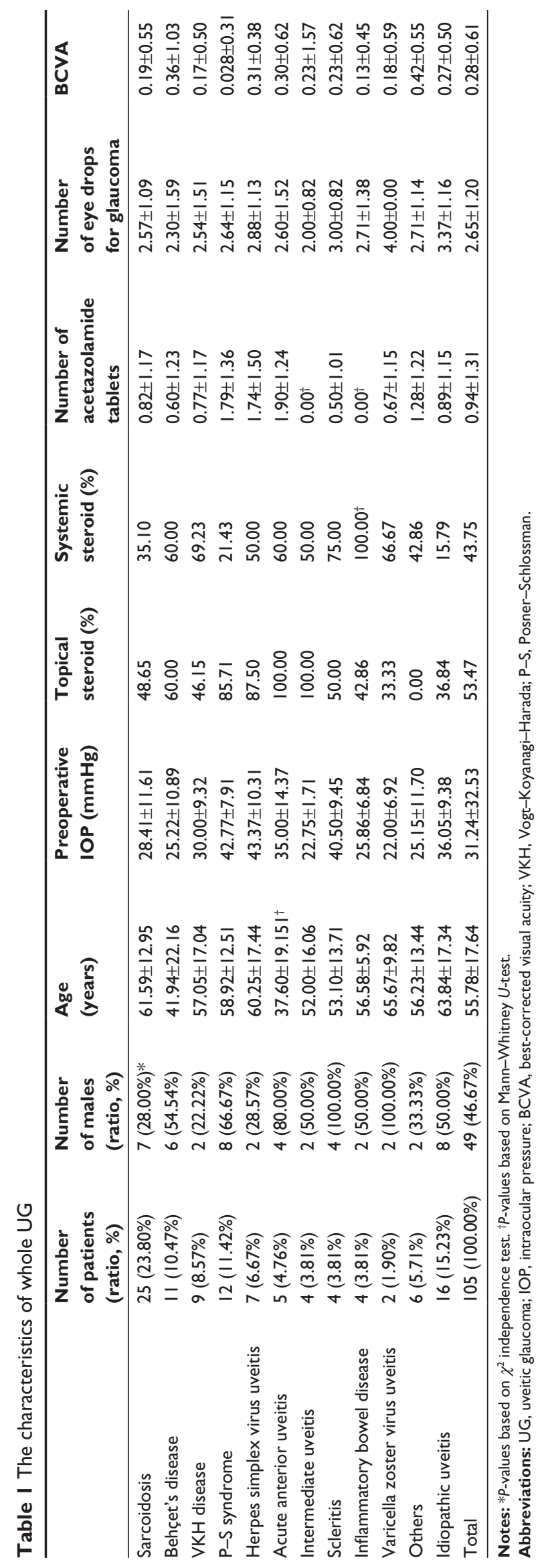


The mean follow-up period was $40.32 \pm 32.53$ months, mean age was $56.20 \pm 17.03$ years, mean pretreatment IOP was $31.24 \pm 32.53 \mathrm{mmHg}$, and mean BCVA was $0.28 \pm 0.61$ $\log$ MAR. Patients with P-S syndrome had the highest BCVA (0.028 \pm 0.31$)$, and patients with Behçet's disease had the worst $(0.36 \pm 1.03)$, with no significant differences in the other groups. Extensive PAS (covering more than half the perimeter of the iris) was observed in 18 patients (17.14\%), nonextensive PAS was observed in 35 patients (33.33\%), and no PAS was observed in 52 patients (49.52\%).

\section{Medical treatment for UG}

The overall consecutive use of topical steroids to treat uveitis in the UG patients was $53.47 \%$, with higher rates in the acute anterior uveitis and herpes simplex virus uveitis patients (100.00\%) and no significant differences in the other groups. By contrast, the overall consecutive usage of systemic steroids was $43.75 \%$, with a significantly higher rate in patients with inflammatory bowel disease $(100.00 \%)(P<0.05)$.

The average number of eye drops prescribed for ocular hypotensive therapy was $2.65 \pm 1.20$, with no significant differences in any group. The average number of acetazolamide tablets used to control IOP for uncontrollable glaucoma by topical treatment was $0.94 \pm 1.31$. Patients with intermediate uveitis and inflammatory bowel disease did not use acetazolamide $(P<0.05)$.

\section{Surgical data analysis}

Forty-seven patients (47 eyes) underwent glaucoma surgery. The mean age of these patients was $58.70 \pm 17.87$ years. Twenty male patients and 27 female patients were included in this study group, including cases of sarcoidosis $(n=9$, $18.75 \%)$, Behçet's disease $(\mathrm{n}=5,10.42 \%)$, VKH disease $(\mathrm{n}=5$, $10.42 \%), \mathrm{P}-\mathrm{S}$ syndrome $(\mathrm{n}=6,12.50 \%)$, herpes simplex virus uveitis $(n=4,8.33 \%)$, acute anterior uveitis $(n=4,8.33 \%)$, idiopathic UG $(n=9,18.75 \%)$, and others $(n=5,10.42 \%)$. Thirty-two patients had granulomatous uveitis $(68.09 \%)$ and 15 patients had nongranulomatous uveitis (31.91\%). The mean pretreatment IOP was $39.09 \pm 10.41 \mathrm{mmHg}$.

Trabeculectomy was performed in 35 patients, trabeculotomy in eight patients, and trabectome surgery in four patients. The mean postoperative follow-up period was $39.46 \pm 37.66$ months and the longest was 160 months. The success rate was $82.86 \%$ for trabeculectomy, $62.50 \%$ for trabeculotomy, and $75.00 \%$ for trabectome surgery, with failure occurring in 10 patients overall. There were 22 successful cases in which eye drops were not prescribed eye drops for ocular hypotensive therapy (46.81\%) and 37 successful cases in which they were prescribed eye drops for ocular hypotensive therapy (78.72\%). The success rate in different types of uveitis was $87.50 \%$ in granulomatous uveitis patients $(\mathrm{n}=28)$ and $60.00 \%$ in nongranulomatous uveitis patients $(n=9)$. The success rate according to the underlying cause of UG was as follows: $100.00 \%$ for VKH disease, $88.89 \%$ for sarcoidosis, $83.33 \%$ for P-S syndrome, $77.78 \%$ for idiopathic UG, $75.00 \%$ for herpes simplex virus uveitis, $50.00 \%$ for acute anterior uveitis, and $40.00 \%$ for Behçet's disease.

\section{Prognostic factors for UG after surgery}

An analysis of potential prognostic factors revealed that male sex $(P=0.02)$, age less than 45 years $(P=0.0009)$, and nongranulomatous uveitis $(P=0.04)$ were significant risk factors for surgical failure (Figure 1A-C). A high number of acetazolamide tablets $(P=0.28)$, a high number of eye drops prescribed for ocular hypotensive therapy $(P=0.16)$, pretreatment IOP more than $30 \mathrm{mmHg}(P=0.07)$, or the use of topical steroids $(P=0.44)$ or systemic steroids $(P=0.31)$ was not significantly correlated with surgical failure. Extensive PAS was observed in 12 patients $(25.53 \%)$, but the success rate was not significantly different in patients with extensive PAS, nonextensive PAS, and no PAS ( $P=0.12$ data not shown).

Next, we determined the influence of preoperative and postoperative intraocular inflammations on IOP control after glaucoma surgery. Preoperatively, 18 patients had inflammation, with aqueous cell scores of $1+(n=17)$ or $2+(n=1)$; and 29 patients had no preoperative inflammation, with a score of 0 or trace. Postoperatively, seven patients had inflammation, with aqueous cell scores of $1+(n=6)$ or $2+(n=1)$, versus 40 patients without inflammation. An analysis revealed that the success rate was not significantly different in the groups with and without preoperative inflammation $(P=0.72$, data not shown), whereas a significant difference was found in the success rate in groups with and without postoperative inflammation ( $P=0.01$, Figure 2).

\section{Postoperative complications}

Postoperative complications included hyphema in four patients $(8.51 \%)$, choroidal detachments in four patients $(8.51 \%)$, and hypotonic maculopathy in four patients (8.51\%). Bleb leakage requiring additional sutures occurred in two eyes (4.26\%), but neither required surgical intervention. One eye $(2.13 \%)$ developed infectious endophthalmitis, and required vitreous surgery. Three eyes (6.38\%) required additional cataract surgery because of postoperative cataract progression. 
A

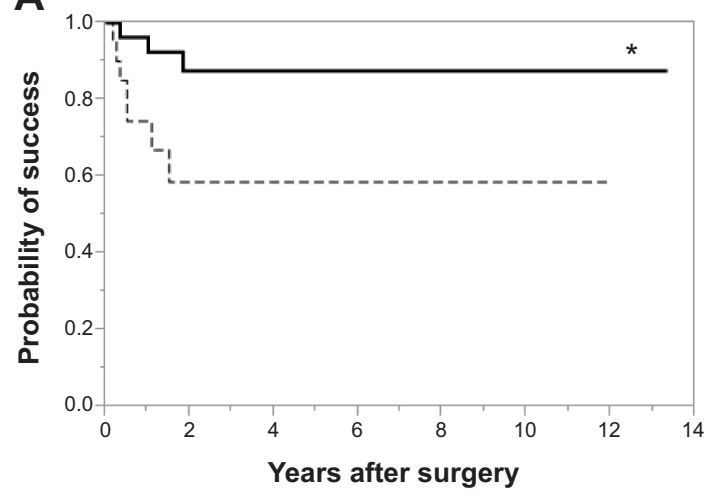

B

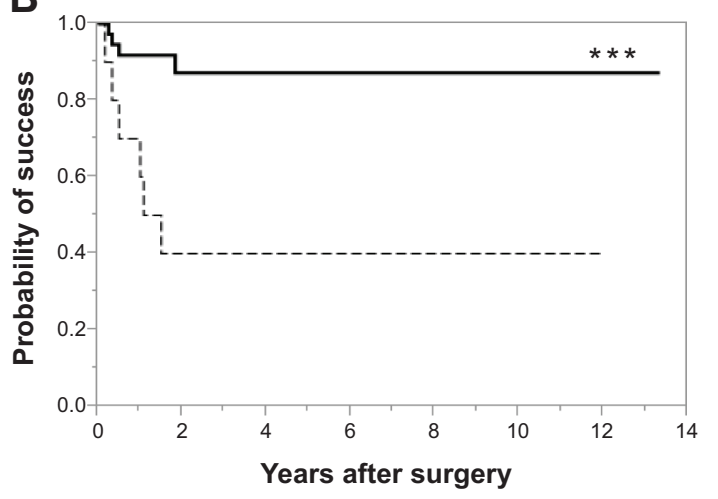

C

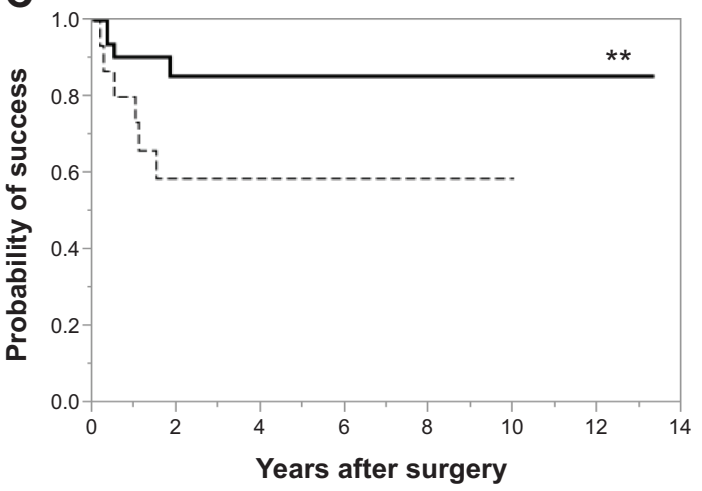

Figure I Kaplan-Meier survival curves of surgical failure in male and female patients, patients younger or older than 45 years, and patients with or without granulomatous uveitis.

Notes: (A) Male ( $n=20$, broken line) versus female ( $n=27$, solid line). $* P=0.02$, log-rank test. (B) Age younger ( $n=10$, broken line) versus older ( $n=37$, solid line) than 45 years. $* * * P=0.0009$, log-rank test. $(\mathbf{C})$ Granulomatous uveitis versus $(n=32$, solid line) versus nongranulomatous uveitis ( $n=15$, broken line). $* * P=0.04$ log-rank test.

\section{Discussion}

In the present study, UG patients comprised $9.73 \%$ of a database of glaucoma patients at our institution. This confirms previous studies reporting that UG comprises $5 \%-15 \%$ of cases of glaucoma. ${ }^{5}$ However, although there have been a small number of reports on the patient characteristics of secondary glaucoma in Japan, ${ }^{2,6}$ whose findings on the

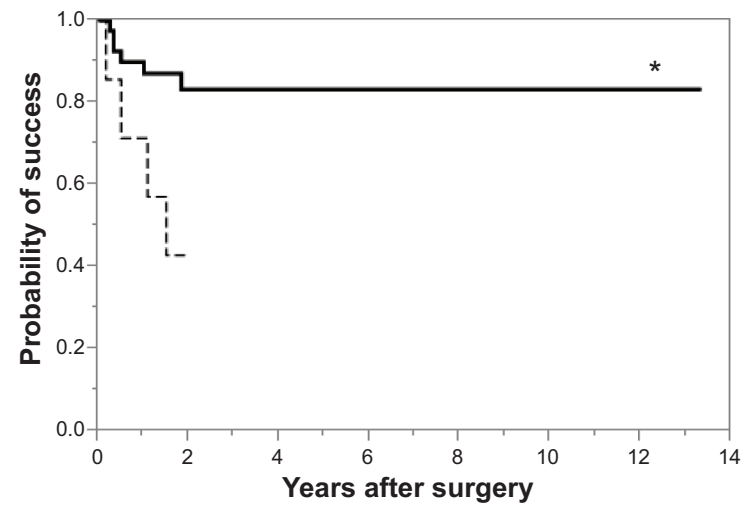

Figure 2 Kaplan-Meier survival curves of surgical failure with $(n=7)$ versus without $(n=40)$ postoperative inflammation.

Note: $* P=0.04$ incidence of secondary glaucoma agree with the current report, there has not yet been any independent report on UG. Therefore, we chose to investigate the incidence of UG, the characteristics of UG patients, and the outcomes of surgical treatment (risk factor for surgical failure) for UG. Our present result indicated that young age, male sex, nongranulomatous uveitis, and the grade of postsurgical inflammation were risk factors for surgical failure.

Previous studies of the incidence of the three major types of uveitis in Japan (sarcoidosis, Behçet's disease, and VKH disease) reported data on the sex distribution of UG patients that agree with our findings. ${ }^{6,11,16}$ Specifically, sarcoidosis occurred more often in the female patients in our study, and P-S syndrome occurred more often in the male patients. However, there was no difference in the frequency of Behçet's disease between male and female patients. ${ }^{16}$ Yang et al ${ }^{17,18}$ reported that VKH disease had no difference in frequency between male and female patients, but found that Behçet's disease occurred more often among male patients in the People's Republic of China. This discrepancy may be because of ethnicity. We also found that the incidence of $\mathrm{P}-\mathrm{S}$ 
syndrome was relatively higher in this study than in previous reports. ${ }^{7,11,19}$ These discrepancies may be because of population variations between UG patients who undergo surgery and uveitis patients overall. Moreover, our study included only a small number of patients with types of uveitis other than the major three types, and we were unable to accurately evaluate these types of the disease. There is therefore a need for a more comprehensive investigation, with a larger study population of different types of uveitis.

Surgical outcomes for the patients in this study were similar to those reported in previous studies, including the rates of surgical success and postoperative complications. ${ }^{11,19,20}$ A number of previous reports found that surgery for UG had at least a $60 \%$ success rate: Kaburaki et al ${ }^{19}$ reported a $64.7 \%$ success rate for trabeculectomy in $53 \mathrm{UG}$ patients, Iwao et $\mathrm{al}^{4,11}$ reported a $71.3 \%$ success rate for trabeculectomy in 121 patients and a $78.1 \%$ success rate for trabeculotomy in 101 patients, Sidoti et $\mathrm{al}^{21}$ reported a $59 \%$ success rate in 29 children, and Minckler et al $^{22,23}$ reported an $84 \%$ success rate for trabectome surgery.

Our study also revealed that young age, male sex, and nongranulomatous uveitis were risk factors for surgical failure. This result is understandable given the nature of uveitis. In particular, this reinforces the finding that the success rate for glaucoma surgery in patients with Behçet's disease was the lowest among the groups. Behçet's disease is a systemic inflammatory disease that does not cause granuloma formation and has higher rates of serious attacks in young men. ${ }^{18,24,25}$ Together, these findings suggest that inflammation affects surgical success. Specifically, our results showed that postoperative inflammation significantly affected surgical success, while preoperative inflammation was not a significant risk factor. Previous reports also suggested that surgical success was affected by postoperative inflammation, but not by preoperative inflammation. ${ }^{19}$ Postoperative fibrosis and inflammation of the trabecular meshwork and conjunctiva have also been suggested as factors affecting the success of filtration surgery. ${ }^{26}$ Multiple inflammatory cytokines, such as interleukin-6, interleukin-8, and monocyte chemoattractant protein-1, can activate fibroblasts; inflammatory leukocytes, such as neutrophils; and macrophage infiltration. In particular, Inoue et al reported that monocyte chemoattractant protein-1 levels in the aqueous humor were a prognostic factor for the outcome of trabeculectomy. ${ }^{27}$ In fact, infiltrating cells increase in the conjunctival filtering bleb in postoperative UG patients. In addition, it has been reported that a large amount of inflammatory cytokines are naturally present in the aqueous humor in active uveitis. Overall, therefore, our study strongly suggests that suppression of postoperative inflammation may be an important approach to improving the outcome of glaucoma operations.

In conclusion, despite being limited by its retrospective design, this study made the novel finding that UG accounted for approximately $10 \%$ of all cases of glaucoma. Moreover, young men with nongranulomatous uveitis had a significantly higher risk of surgical failure. Finally, we found that prolonged postoperative inflammation also created a higher risk of surgical failure, suggesting that inflammation reduction after surgery may be an important treatment.

\section{Limitations of this study}

UG also includes steroid-induced glaucoma. In the present study, we focused on patient with ocular hypertension and a background of uveitis. Further investigations should therefore be performed in the near future.

\section{Acknowledgment}

We would like to thank Mr Tim Hilts for editing and critical reading of this manuscript.

\section{Author contributions}

All authors contributed to the study and to the manuscript preparation, and reviewed and approved the manuscript.

\section{Disclosure}

The authors report no conflicts of interest in this work.

\section{References}

1. Merayo-Lloves J, Power WJ, Rodriguez A, Pedroza-Seres M, Foster CS. Secondary glaucoma in patients with uveitis. Ophthalmologica. 1999;213(5):300-304.

2. Takahashi T, Ohtani S, Miyata K, Miyata N, Shirato S, Mochizuki M. A clinical evaluation of uveitis-associated secondary glaucoma. Jpn J Ophthalmol. 2002;46(5):556-562.

3. Herbert HM, Viswanathan A, Jackson H, Lightman SL. Risk factors for elevated intraocular pressure in uveitis. J Glaucoma. 2004;13(2):96-99.

4. Iwao K, Inatani M, Tanihara H. Success rates of trabeculotomy for steroid-induced glaucoma: a comparative, multicenter, retrospective cohort study. Am J Ophthalmol. 2011;151(6):1047-1056; e1041.

5. Neri P, Azuara-Blanco A, Forrester JV. Incidence of glaucoma in patients with uveitis. J Glaucoma. 2004;13(6):461-465.

6. Yamamoto T, Iwase A, Araie M, et al. The Tajimi Study report 2: prevalence of primary angle closure and secondary glaucoma in a Japanese population. Ophthalmology. 2005;112(10):1661-1669.

7. Kanda T, Shibata M, Taguchi M, Ishikawa S, Harimoto K, Takeuchi M. Prevalence and aetiology of ocular hypertension in acute and chronic uveitis. Br J Ophthalmol. 2014;98(7):932-936.

8. The Japan Glaucoma Society Guidelines for Glaucoma (3rd Edition). Nihon Ganka Gakkai Zasshi. 2012;116(1):3-46.

9. Bloch-Michel E, Nussenblatt R. International Uveitis Study Group recommendations for the evaluation of intraocular inflammatory disease. Am J Ophthalmol. 1987;103(2):234-235. 
10. Jabs D, Nussenblatt R, Rosenbaum J. Standardization of uveitis nomenclature for reporting clinical data. Results of the First International Workshop. Am J Ophthalmol. 2005;140(3):509-516.

11. Iwao K, Inatani M, Seto T, et al. Long-term outcomes and prognostic factors for trabeculectomy with mitomycin $\mathrm{C}$ in eyes with uveitic glaucoma: a retrospective cohort study. J Glaucoma. 2014;23(2):88-94.

12. Nussenblatt RB, Whitcup SM, Palestine A. Uveitis: Fundamentals and Clinical Practice. Philadelphia: Mosby; 2004.

13. Cairns J. Trabeculectomy-preliminary report of a new method. Am J Ophthalmol. 1968;66:673-679.

14. Kitazawa Y, Kawase K, Matsushita H, Minobe M. Trabeculectomy with mitomycin: a comparative study with fluorouracil. Arch Ophthalmol. 1991;109(12):1693-1698.

15. Ellingsen BA, Grant WM. Trabeculotomy and sinusotomy in enucleated human eyes. Invest Ophthalmol Vis Sci. 1972;11(1):21-28.

16. Ohguro N, Sonoda K-H, Takeuchi M, Matsumura M, Mochizuki M. The 2009 prospective multi-center epidemiologic survey of uveitis in Japan. Jpn J Ophthalmol. 2012;56(5):432-435.

17. Yang P, Ren Y, Li B, Fang W, Meng Q, Kijlstra A. Clinical characteristics of Vogt-Koyanagi-Harada syndrome in Chinese patients. Ophthalmology. 2007;114(3):606-614; e603.

18. Yang P, Fang W, Meng Q, Ren Y, Xing L, Kijlstra A. Clinical features of Chinese patients with Behçet's disease. Ophthalmology. 2008;115(2):312-318; e314.
19. Kaburaki T, Koshino T, Kawashima H, et al. Initial trabeculectomy with mitomycin $\mathrm{C}$ in eyes with uveitic glaucoma with inactive uveitis. Eye. 2009;23(7):1509-1517.

20. Ceballos EM, Beck AD, Lynn MJ. Trabeculectomy with antiproliferative agents in uveitic glaucoma. J Glaucoma. 2002;11(3):189-196.

21. Sidoti PA, Belmonte SJ, Liebmann JM, Ritch R. Trabeculectomy with mitomycin-C in the treatment of pediatric glaucomas. Ophthalmology. 2000;107(3):422-429.

22. Minckler DS, Baerveldt G, Alfaro MR, Francis BA. Clinical results with the Trabectome for treatment of open-angle glaucoma. Ophthalmology. 2005;112(6):962-967.

23. Minckler D, Mosaed S, Dustin L, Francis B, Group TS. Trabectome (trabeculectomy - internal approach): additional experience and extended follow-up. Trans Am Ophthalmol Soc. 2008;106:149.

24. Elgin U, Berker N, Batman A. Incidence of secondary glaucoma in Behcet disease. J Glaucoma. 2004;13(6):441-444.

25. Park J, Han M, Bettmann M. Arterial manifestations of Behcet disease. Am J Roentgenol. 1984;143(4):821-825.

26. Baudouin C, Pisella P-J, Fillacier K, et al. Ocular surface inflammatory changes induced by topical antiglaucoma drugs: human and animal studies. Ophthalmology. 1999;106(3):556-563.

27. Inoue $\mathrm{T}$, Kawaji $\mathrm{T}$, Tanihara H. Monocyte chemotactic protein-1 level in the aqueous humour as a prognostic factor for the outcome of trabeculectomy. Clin Experiment Ophthalmol. 2014;42(4):334-341.
Clinical Ophthalmology

\section{Publish your work in this journal}

Clinical Ophthalmology is an international, peer-reviewed journa covering all subspecialties within ophthalmology. Key topics include: Optometry; Visual science; Pharmacology and drug therapy in eye diseases; Basic Sciences; Primary and Secondary eye care; Patien Safety and Quality of Care Improvements. This journal is indexed on

Submit your manuscript here: http://www.dovepress.com/clinical-ophthalmology-journal

\section{Dovepress}

PubMed Central and CAS, and is the official journal of The Society of Clinical Ophthalmology (SCO). The manuscript management system is completely online and includes a very quick and fair peer-review system, which is all easy to use. Visit http://www.dovepress.com/ testimonials.php to read real quotes from published authors. 